\title{
A New Combined Model for considering the Plasticity Effects in Contacting Asperities
}

\author{
Mohammad Amini Sarabi $(\mathbb{D})$ and Parisa Hosseini Tehrani \\ School of Railway Engineering, Iran University of Science and Technology, Tehran 16846-13114, Iran \\ Correspondence should be addressed to Parisa Hosseini Tehrani; hosseini_t@iust.ac.ir
}

Received 11 August 2020; Revised 18 October 2020; Accepted 8 November 2020; Published 18 November 2020

Academic Editor: Denis Benasciutti

Copyright ( $) 2020$ Mohammad Amini Sarabi and Parisa Hosseini Tehrani. This is an open access article distributed under the Creative Commons Attribution License, which permits unrestricted use, distribution, and reproduction in any medium, provided the original work is properly cited.

\begin{abstract}
Wheel-rail contact in railway engineering is an important topic. Due to different materials and surface roughness of wheel and rail, the contact characteristics can alter significantly. This article aims to investigate the effects of surface roughness and asperities on the contact parameters such as contact area, contact force, and contact stiffness. The lateral contacts between asperities are assumed to be the general contact condition. Azimuthal and contact angles distributions are assumed to be spherical harmonic distribution. This assumption is compatible with the asperity distribution on the wheel and the rail surfaces. Besides, a new combined model is developed to cover the stick-slip and the plasticity effects in contacting asperities. The results of the presented model offer very good estimations for the asperities contact characteristics, especially at the small-contact area and separation where high-contact pressure and plastic deformation usually exist.
\end{abstract}

\section{Introduction}

Nowadays, railway engineering has enormously developed. However, many unidentified subjects regarding wheel-rail failure and maintenance cycles need to be investigated yet [1]. Contact of surfaces is a high-risk matter from the damage initiation point of view in engineering problems and the railway industry. To develop the accuracy of the contact modeling, the topography classification of the surfaces is a real need. To this end, probabilistic models are presented to describe the contact characteristics like contact load, contact area, separation of surfaces, and contact stiffness. Furthermore, the contact stiffness may alter according to the stated features. These features usually cause complicated and nonlinear problems in studying the contacting surfaces $[2,3]$. To improve the modeling of contacting rough surfaces, the effects of the asperities should be taken into account. In this regard, the following applied models are introduced.

Greenwood and Williamson [4] defined the roughness distribution by considering the spherical asperities with different heights on the surface and introduced the GW model in terms of the Hertz theory for elastic contact. To spread the GW model to a fully plastic zone, Chang et al. [5] suggested their model named as CEB. The ZMC model is introduced by Zhao et al. [6] to evaluate the deformation of asperities in the elastic-plastic regime. In this regard, they examined the mathematical fitting approach. In [7], the elastic-plastic asperity deformation in contacting surfaces by using FEM is investigated by Kogut and Etsion. Their approach is known as the KE model.

In [8], Etsion used another FE model to examine the dependence of load to deformation and force considering plasticity in contact problems. In [9], Jackson and Green consider the effects of hardness variation by a FE analysis (JG Model). They examined elastic-plastic contact of a sphere with a rigid flat surface considering geometrical hardness variation. The mentioned models are constructed in terms of the idea of contacting an equal rough surface with a rigid one without roughness. As a result, the stick-slip phenomenon at the asperities shoulder may not be considered by these models [10]. In [11], Ciavarella used an uncomplicated model for contacting rough surfaces. However, his model does not show good accuracy because of the assumptions 
and simplifications in many parameters in contact problems that impose a significant effect on the final results.

Cinat et al. [12] proposed an algorithm that exploited an analogy between genetics and the multiscale characterization of roughness, where various-length scales are described in terms of rough profiles. Wen et al. [13] studied a new elliptical microcontact model considering elastoplastic deformation. They described the relationship between the normal deformation of the asperity and the mean contact pressure.

Some other works like Abuzeid et al. [14] have used fractal methods for estimating the properties of the rough surface contact. This method is different from the statistical model and usually is adequate for accounting based on frequencies.

Lately, Megalingam and Hanumanth Ramji [15] proposed a model of a deformable spherical asperity contact with a rigid flat plate in a statistical method and combined their model with the effects of Young's modulus, Poisson's ratio, yield strength, and isotropic strain hardening rate by using the finite element method.

Abdo and Farhang [16] suggested the AF model for contacting of the rough surfaces by considering shoulder-toshoulder asperity contact and plasticity. They considered a distinct condition among the different contact zones.

In [5], Chang et al. at the macroscopic level used an elastic-plastic model while at the microscopic scale, they considered asperities experience initially elastic deformation, and where their defined criterion had been met, they assumed purely plastic deformation.

In recent studies, the used models almost consider the elastic-plastic behavior of the materials $[17,18]$.

In [19], Gao et al. gave an elastic-plastic contact model for contacting asperities. They assumed the lateral contact, effects of adjacent asperities, and distribution of contact angles. Although their research is the closest work to the presented study, they did not consider the effects of the stickslip in the modeling of contacting asperities, and they used a rather complicated model for implying the effects of contact angles.

According to the performed analysis by using the conventional models, there are still many lacks in the contact modeling of asperities. For example, most of the existing models assume that an equal rough surface contact with a rigid flat one while the effects of friction and interaction between adjacent asperities are passed over. Besides, many models consider isotropic features for a rough surface that may be unrealistic.

In this task, a new combined model is constructed to study the contacting asperities. By this model, the lateral contact between asperities and the elastic-plastic regime in the contact area are considered. Using the base of the AF model [16] for the elastic-plastic regime, focus on the slipstick in the asperity contact region is done. Furthermore, a new spherical harmonic function is developed for asperity contact modeling that its usage shows more precision in comparison with the experimental results.

For a better understanding, the characteristics and main fields of application of different well-known models are shown in Table 1.

\section{Methods}

In this research, based on Gao et al.'s works [19], contact of two single asperities under shoulder-to-shoulder interaction is considered. The model of contacting asperity is demonstrated in Figure 1, where the mean separation $d$ is the gap between mean planes 1 and 2 . The mean plane represents the mean value of asperity height distribution on each surface.

In this task, to avoid the complexity in formulations, the shape of the asperity's tip was assumed to be spherical.

Abdo and Farhang [16] defined a fictitious asperity with a fictitious surface which is related to the plastic deformation of asperity (Figure 1). As is shown in Figure $1, \omega_{c}$ represents the critical deformation at the beginning of plastic deformation and $R$ represents the radius of the spherical tip of asperity. By analyzing the contact at the tip of the asperities, according to Figure 2, the contact force relationships can be found.

The total contact force decomposes into three components as follows:

$$
\begin{aligned}
& F_{i n}=F_{i} \cos \varphi, \\
& F_{i \tau}=F_{i} \sin \varphi \sin \theta, \\
& F_{i s}=F_{i} \sin \varphi \cos \theta,
\end{aligned}
$$

where $\varphi$ and $\theta$ are contact and azimuthal angles, respectively. The resultant force $F_{i}$ is calculated as follows:

$$
F_{i}=\sqrt{F_{i n}^{2}+F_{i \tau}^{2}+F_{i s}^{2}}
$$

The normal component force $F_{\text {in }}$ makes a relating normal deformation $\delta_{b}$ in the $Z^{\prime}$-axis direction which includes the deformation $\delta_{b f}$ (as shown in Figure 2) produced due to the lateral contact of lower and upper asperities and the deformation $\delta_{b w}$ created due to contact of the nearby asperities. The normal component of deformation $\delta_{b}$ is shown as follows:

$$
\delta_{b}=\delta_{b f}+\delta_{b w}
$$

The tangential component of force $F_{i \tau}$ creates a tangential displacement in the $X^{\prime}$-axis direction, and the horizontal component of force $F_{\text {is }}$ generates a horizontal displacement in the $Y^{\prime}$-axis direction.

Considering the elastic-plastic model of material, Gao et al [19] introduced the relations of force, contact area, and contact stiffness between contacting asperities. They considered interactional deformation effects of adjacent asperities and anisotropic spreading of asperities. In this work based on [7], [16], and [19], we analysed the elastic-plastic contact regime by considering a new combined model for the asperities contact problem. The presented model is accurately compatible with the physics of the asperities contact problem and the results of the experiment. To examine the validity of the presented method, the obtained results are compared with the results of the other related works.

2.1. Analysis of the New Combined Method. In [7], Kogut and Etsion solved the problem of elastic-plastic contacting asperities and noted that the complete elastic-plastic state 
TABLE 1: Different important models in rough surfaces contact problem.

\begin{tabular}{|c|c|c|}
\hline Model & Key characteristics & Main fields of application \\
\hline $\begin{array}{l}\text { GW model (Greenwood } \\
\text { and Williamson) }\end{array}$ & Considering the spherical asperities & The elastic contact based on Hertz theory \\
\hline $\begin{array}{l}\text { CEB model (Chang, Etsion, } \\
\text { and Bogy) }\end{array}$ & Considering plastic effects & $\begin{array}{l}\text { The elastic- and fully-plastic zones in } \\
\text { rough surface contact }\end{array}$ \\
\hline $\begin{array}{l}\text { ZMC model (Zhao, Maietta, } \\
\text { and Chang) }\end{array}$ & Evaluating the deformation of asperities & $\begin{array}{l}\text { The elastic-plastic contact in rough } \\
\text { surfaces }\end{array}$ \\
\hline $\begin{array}{l}\text { KE model (Kogut and } \\
\text { Etsion) }\end{array}$ & FEM for elastic-plastic asperity deformations & $\begin{array}{c}\text { The elastic-plastic contact in rough } \\
\text { surfaces (contact between asperity and a } \\
\text { flat surface) }\end{array}$ \\
\hline Etsion model & $\begin{array}{l}\text { FE model to examine the dependence of load to deformation } \\
\text { and force }\end{array}$ & $\begin{array}{l}\text { The elastic- and perfect-plastic contact in } \\
\text { rough surfaces }\end{array}$ \\
\hline $\begin{array}{l}\text { JG model (Jackson and } \\
\text { Green) }\end{array}$ & Considering the effects of hardness using the FE method & $\begin{array}{c}\text { The elastic-plastic contact in rough } \\
\text { surfaces (contact between asperity and a } \\
\text { flat surface) }\end{array}$ \\
\hline $\begin{array}{l}\text { AF model (Abdo and } \\
\text { Farhang) }\end{array}$ & Considering shoulder-to-shoulder asperity contact & $\begin{array}{l}\text { The elastic-plastic contact in rough } \\
\text { surfaces }\end{array}$ \\
\hline Gao model (Gao et al.) & $\begin{array}{c}\text { Considering the lateral contact, effects of adjacent asperities, } \\
\text { and distribution of contact angles }\end{array}$ & $\begin{array}{l}\text { The elastic-plastic contact in rough } \\
\text { surfaces }\end{array}$ \\
\hline $\begin{array}{l}\text { Present model or AT model } \\
\text { (Amini and Tehrani) }\end{array}$ & $\begin{array}{c}\text { Considering the lateral contact, effects of adjacent asperities, } \\
\text { distribution of contact angles, slip-stick effects, and } \\
\text { multiasperities contact }\end{array}$ & $\begin{array}{l}\text { The elastic-plastic contact in rough } \\
\text { surfaces }\end{array}$ \\
\hline
\end{tabular}

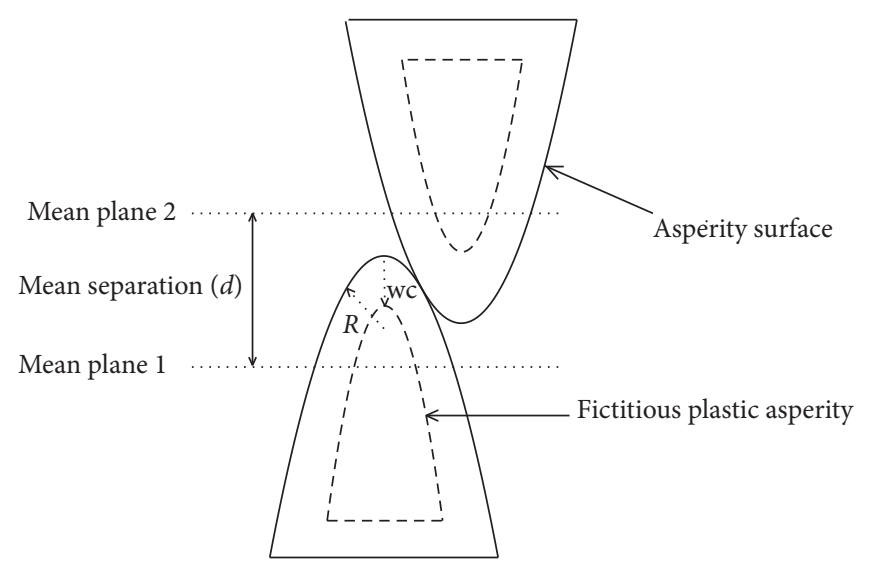

Figure 1: Model of contacting asperities under shoulder-to-shoulder interaction.
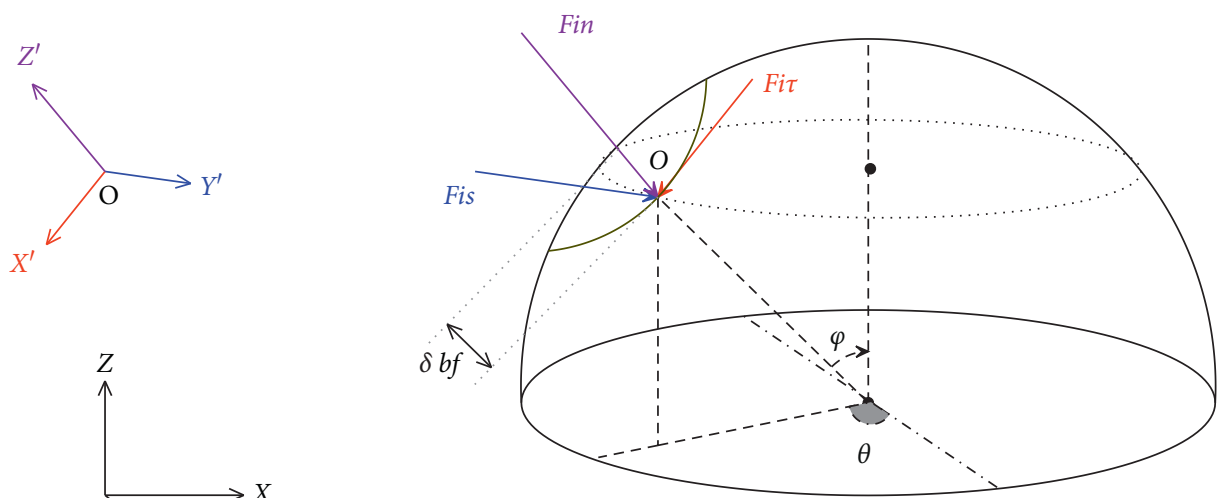

FIGURE 2: Asperities contact and force analysis. 
spreads over interference values in the range of $1 \leq \delta_{b f} f$ $\omega_{c}<110$ with a marked variation in the mean contact pressure at $\delta_{b f} / \omega_{c}=6$. Up to the $\delta_{b f} / \omega_{c}=6$, which is a shifting value; a plastic zone grows under the contact boundary while the whole contact area is elastic. Above $\delta_{b f} / \omega_{c}=6$, the contact area includes an internal elastic circular core that is enclosed by an outer plastic annulus.

Gao et al. [19] reported that the stick area is a circular core at the elastic-plastic state and is bounded by an outer plastic zone that is based on Cattaneo [20], Mindlin [21], and Eriten et al.'s [22] works; we can mention this region as the partial-slip area (Figure 3).

According to Kogut and Etsion's [7] model, the whole area of the contact is plastic, but the mean contact pressure stays growing until it turns out to be unvarying and equal to the hardness at $\delta_{b f} / \omega_{c}=110$, where the fully plastic contact arises.

Considering the three general regimes elastic, elasticplastic, and entirely plastic deformations for asperities under contact, Gao et al. [19] showed that the total force, contact area, and stiffness are obtained as follows (if we assume that $Q$ is a property like a force or stiffness of asperities in contact):

$$
Q_{T O T}=Q_{e}+Q_{e p}+Q_{p},
$$

where $Q_{T O T}, Q_{e}, Q_{e p}$, and $Q_{p}$ are the total property of asperities in contact, the property related to elastic regime, the property related to elastic-plastic regime, and the property related to the fully plastic regime, respectively.

Therefore, based on the Kogut and Etsion's [7] and Abdo and Farhang's [16] models and Gao et al.'s [19] works, we can rewrite equation (4) as follows:

$$
Q_{T O T}=Q_{e}-Q_{e p 1}+Q_{e p 2}+Q_{p} \text {, }
$$

where $Q_{e p 1}$ is the elastic-plastic first component of the property that is correlated to the stick area, and in terms of the Abdo and Farhang's [16] model, it should be subtracted from the elastic property and $Q_{e p 2}$ is the elastic-plastic second component of the property that is related to the slip area, and based on the Gao et al. [19], the formulation should be added with the other regimes. In this analysis, we can consider both slip and stick effects in the elastic-plastic zone and modify the elastic-plastic formulations in the contact problem of asperities. The effects of fictitious asperity (AF model) may be considered in relations as follows:

$$
R_{p}=\left(R-\omega_{c}\right)
$$

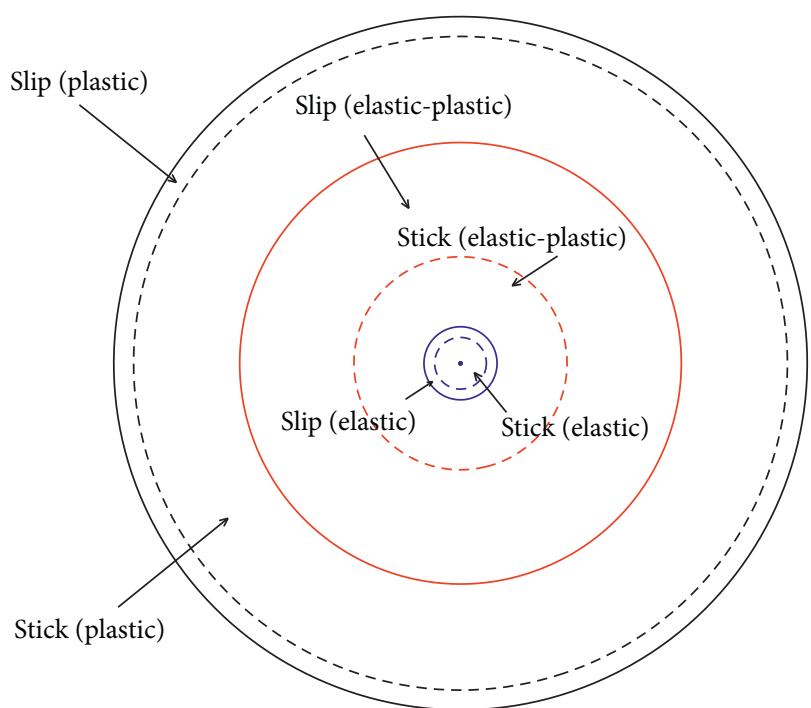

Figure 3: The areas of the slip and stick.

where $R$ is the equivalent radius of asperities, $R_{p}$ is the equivalent plastic radius based on the AF model [16], and $\omega_{c}$ is the critical deformation at the beginning of plastic state. According to the CEB model [5] the relations for these parameters can be expressed as follows:

$$
\begin{aligned}
R & =\left(R_{1}^{-1}-R_{2}^{-1}\right)^{-1}, \\
\omega_{c} & =\left(\frac{\pi S H}{2 E}\right)^{2} R,
\end{aligned}
$$

where $R_{1}$ and $R_{2}$ are the radii of the asperities near the contact zone and $S$ is the maximum contact pressure factor, which is based on the CEB model [5] obtained by $S=0.454+0.41 v, v$ and $H$ are Poisson's ratio and the hardness of the softer material, respectively, and $E$ is the equivalent elastic modulus which is attained as follows:

$$
E=\left(E_{1}^{-1}-E_{2}^{-1}\right)^{-1}
$$

2.2. Harmonic Spherical Distribution Function for Effects of Contact Angles. In this work, we have chosen a new harmonic spherical distribution function to reveal the effects of shoulder-to-shoulder contact angles. Poorsolhjouy and Gonzalez [23] introduced a new distribution function $\psi(\varphi, \theta)$ for asperities contact as follows:

$$
\psi(\varphi, \theta)=\frac{1}{4 \pi}\left\{1+\sum_{k=2}^{\infty}\left(a_{k 0} P_{k}(\cos \varphi)+\sum_{m=1}^{\infty} P_{k}^{m}(\cos \varphi)\left(a_{k m} \cos m \theta+b_{k m} \sin m \theta\right)\right)\right\}
$$

where $\varphi$ and $\theta$ are contact and azimuthal angles, respectively. The summation over even $k$ is represented by $\Sigma^{\prime} \cdot P_{k}(\cos \varphi)$ is the $k_{t h}$ order Legendre polynomial with respect to $\cos \varphi$, while $P_{k}^{m}(\cos \varphi)$ is its $m_{t h}$ associated Legendre function. Parameters $a_{k 0}, a_{k m}$, and $b_{k m}$ are fabric factors controlling the form of the distribution function. The shape of Legendre 
polynomials $P_{2}$ and $P_{2}^{1}$ are matched to the single asperity contact problem which we assumed at the beginning to simplify equation (9). According to Poorsolhjouy and Gonzalez [23], parameter $a 20$ is equal to -0.5 in a wide range of density values. Furthermore, we assume parameters $a_{21}$ and $b_{21}$ are equal to 1 because the shape of the distribution function for the azimuthal angles is harmonic in the asperities contact zone. Equation (9) can be simplified with these assumptions as follows:

$$
\begin{aligned}
\psi(\varphi, \theta)= & \frac{1}{4 \pi}\left\{1-\frac{1}{4}\left(3 \cos ^{2} \varphi-1\right)\right. \\
& -3(\cos \varphi \cdot \sin \varphi)(\cos \theta+\sin \theta)\} .
\end{aligned}
$$

The distribution function $\psi$, used in asperities contact formulation, states the effects of the contact angle distribution in the final formulation. The variations of $\varphi$ and $\theta$ during integration are 0 to $\pi$ and 0 to $2 \pi$, respectively.

2.3. Final Contact Formulations. According to the stated theories and combining them for elastic-plastic interaction zone and based on [19], the AF model [16], CEB model [5], and KE model [7], the relations for contact force, contact area, and contact stiffness may be computed as follows:

$$
F_{t o t}=F_{e}-F_{e p 1}+F_{e p 2}+F_{p}
$$

where

$$
\begin{gathered}
F_{e}=\eta A_{n} \int_{d^{*}}^{d^{*}+\delta_{b c}^{*}} \int_{0}^{2 \pi} \int_{0}^{\pi} F_{i e}^{*} \sin \varphi \psi(\varphi, \theta) \phi^{*}\left(z^{*}\right) \mathrm{d} \varphi \mathrm{d} \theta \mathrm{d} z, \\
F_{e p 1}=\eta A_{n} \int_{d^{*}+\delta_{b c}^{*}}^{d^{*}+6 \delta_{b c}^{*}} \int_{0}^{2 \pi} \int_{0}^{\pi} F_{i e p 1}^{*} \sin \varphi \psi(\varphi, \theta) \phi^{*}\left(z^{*}\right) \mathrm{d} \varphi \mathrm{d} \theta \mathrm{d} z, \\
F_{e p 2}=\eta A_{n} \int_{d^{*}+6 \delta_{b c}^{*}}^{d^{*}+110 \delta_{b c}^{*}} \int_{0}^{2 \pi} \int_{0}^{\pi} F_{i e p 2}^{*} \sin \varphi \psi(\varphi, \theta) \phi^{*}\left(z^{*}\right) \mathrm{d} \varphi \mathrm{d} \theta \mathrm{d} z, \\
F_{p}=\eta A_{n} \int_{d^{*}+110 \delta_{b c}^{*}}^{\infty} \int_{0}^{2 \pi} \int_{0}^{\pi} F_{i p}^{*} \sin \varphi \psi(\varphi, \theta) \phi^{*}\left(z^{*}\right) \mathrm{d} \varphi \mathrm{d} \theta \mathrm{d} z .
\end{gathered}
$$

The nondimensional deformation parameter $\delta_{b c}^{*}$ is introduced as $\delta_{b c}^{*}=\delta_{b f} / \omega_{c}$. The distribution function of Gauss in nondimensional form [9] may be attained as follows:

$$
\phi^{*}\left(z^{*}\right)=(2 \pi)^{-1}\left(\frac{\sigma}{\sigma_{z}}\right) \exp \left(-\left(\frac{\sigma}{\sigma_{z}}\right)^{2}\left(\frac{z^{* 2}}{2}\right)\right)
$$

in equations (12)-(16) $\eta, A_{n}, z, d, \sigma, \sigma_{z}$, and * are the area density of asperities, nominal contact area, height of asperity calculated from the mean asperity height, mean separation of two rough surfaces, standard deviation of the surface heights distribution, standard deviation of asperity heights distribution, and nondimensional parameters, respectively.

Gao et al. [19] obtained the relations for $F_{i e}^{*}, F_{i e p 2}^{*}$, and $F_{i p}^{*}$ which are used in equations (12)-(15). In this task, considering the same relations for $F_{i e}^{*}, F_{i e p 2}^{*}$, and $F_{i p}^{*}$, we have obtained a new expression for $F_{i e p 1}^{*}$ based on Eriten's model [22] and combined it with the equation (11). The main formulations are as follows:

$$
F_{i e}^{*}=\sqrt{\left(\delta_{v f}^{*} \cos \varphi\right)^{3}+\left(\frac{3}{2} r_{i e}^{*} \zeta_{v}^{*} \sin \varphi \sin \theta\right)^{2}+\left(\frac{3}{2} r_{i e}^{*} \xi_{v}^{*} \sin \varphi \cos \theta\right)^{2}}
$$

where $\delta_{v f}^{*}$ is the nondimensional normal component of deformation in the $Z$-axis direction that is produced by the normal component of force, $\zeta_{v}^{*}$ and $\xi_{v}^{*}$ are nondimensional tangential and horizontal component of displacement in the $Z$-axis direction, respectively, and $r_{i e}^{*}$ is the nondimensional elastic radius of the contact area between the asperities in contact and is defined as $r_{i e}^{*}=\sqrt{\delta_{b c}^{*} / \pi}$. The relation between $\delta_{v f}^{*}$ and $\delta_{b c}^{*}$ is $\delta_{b c}^{*}=\delta_{v f}^{*} \cos \varphi$.

For elastic-plastic and fully plastic regimes according to Section 3 and the AF model [16], the nondimensional radius of the contact area can be considered as follows:

$$
r_{i(p) A T}^{*}=\frac{r_{i(p)}-\omega_{c}}{r_{i c}}=\frac{r_{i(p)}}{r_{i c}}-\frac{\omega_{c}}{r_{i c}}=r_{i(p)}^{*}-\frac{\omega_{c}}{\sqrt{\omega_{c} R}}=r_{i(p)}^{*}-\sqrt{\frac{\omega_{c}}{R}},
$$

where $r_{i(p) A T}^{*}$ is the nondimensional new radius of contact area at plastic zones, $r_{i(p)}$ is the plastic or elastic-plastic radius of contact area, $r_{i c}$ is the critical radius at the beginning of plastic deformation, and $r_{i c}=\sqrt{\omega_{c} R}$. The 
subscript $(p)$ can be "ep" or " $p$ " for elastic-plastic or plastic deformations, respectively. Then, we have

$$
F_{i e p 1}^{*}=\sqrt{\left(1.32\left(\delta_{v f}^{*} \cos \varphi-1\right)^{1.27}+1\right)^{2}+\left(\frac{3}{2} r_{i e p A T}^{*} \zeta_{v}^{*} \sin \varphi \sin \theta\right)^{2}+\left(\frac{3}{2} r_{i e p A T}^{*} \xi_{v}^{*} \sin \varphi \cos \theta\right)^{2}}
$$

where

$$
r_{i e p A T}^{*}=\sqrt{\frac{1.19\left(\delta_{v f}^{*} \cos \varphi-1\right)^{1.1}}{\pi}}-\sqrt{\frac{\omega_{c}}{R}},
$$

and the relations for nondimensional tangential and horizontal displacement are

$$
\begin{aligned}
& \zeta_{v}^{*}=\frac{16 G r_{i c} \zeta_{v}}{3 F_{i n c}}, \\
& \xi_{v}^{*}=\frac{16 G r_{i c} \xi_{v}}{3 F_{i n c}}
\end{aligned}
$$

where $G$ is the equivalent shear modulus, $G=\left(2-v_{1} /\right.$ $\left.G_{1}+2-v_{2} / G_{2}\right)^{-1}$, and $F_{i n c}$ is the critical value at the beginning of the plastic deformation that may be found as $F_{\text {inc }}=4 E R^{1 / 2} \omega_{c}^{3 / 2} / 3$.

According to the above corrections in the radius of the contact area, we can write

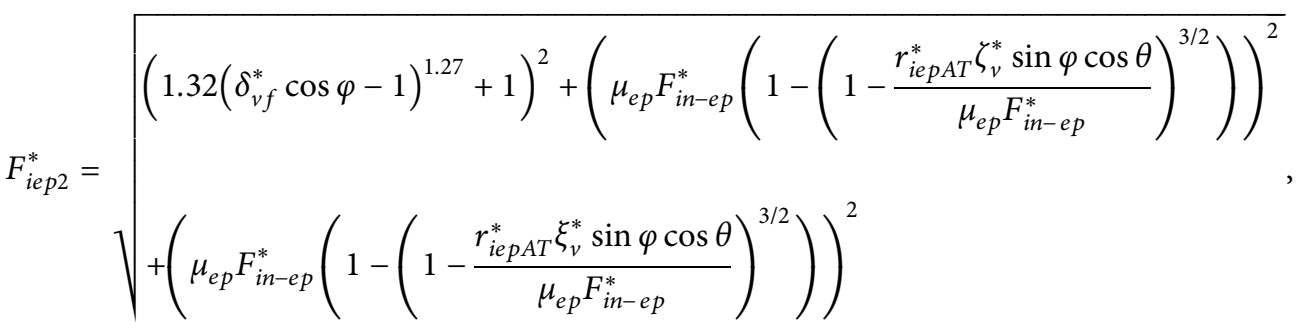

$$
\begin{aligned}
& F_{i p}^{*}=\sqrt{\left(\frac{3 \delta_{v f}^{*} \cos \varphi}{S}\right)^{2}+\left(\frac{3}{2} r_{i p A T}^{*} \zeta_{v}^{*} \sin \varphi \sin \theta\right)^{2}+\left(\frac{3}{2} r_{i p A T}^{*} \xi_{v}^{*} \sin \varphi \cos \theta\right)^{2}},
\end{aligned}
$$

where, according to Etsion's model [12], $F_{i n-e p}^{*}=1.32$ $\left(\delta_{v f}^{*} \cos \varphi-1\right)^{1.27}+1$ and $\mu_{e p}$ is the friction factor at elasticplastic contact deformations, and based on Jaeger's model [24] and CEB model [5], it can be written as $\mu_{e p}=0.27 \operatorname{coth}\left(0.27\left(F_{i n-e p}^{*}\right)^{0.35}\right)$.

According to the JG model [6] and radius corrections in this work,

$$
r_{i p A T}^{*}=\sqrt{\frac{\delta_{v f}^{*} \cos \varphi\left(\delta_{v f}^{*} \cos \varphi / 1.9\right)^{B}}{\pi}}-\sqrt{\frac{\omega_{c}}{R}}
$$

and $B=0.14 \exp (23 S y / E)$, and $S y=(H / 2.8)$.

Finally, we can apply relations (16) to (25) in equations (12) to (15) and using the relation (11) for total asperities contact force.

Similarly, the formulations for total contact area can be written as follows:

$$
A_{\text {tot }}=A_{e}-A_{e p 1}+A_{e p 2}+A_{p}
$$

where 


$$
\begin{aligned}
A_{e} & =\pi \beta \int_{d^{*}}^{d^{*}+\delta_{b c}^{*}} \int_{0}^{2 \pi} \int_{0}^{\pi} A_{i e}^{*} \sin \varphi \psi(\varphi, \theta) \phi^{*}\left(z^{*}\right) \mathrm{d} \varphi \mathrm{d} \theta \mathrm{d} z, \\
A_{e p 1} & =\pi \beta_{p} \int_{d^{*}+\delta_{b c}^{*}}^{d^{*}+6 \delta_{b c}^{*}} \int_{0}^{2 \pi} \int_{0}^{\pi} A_{i e p 1}^{*} \sin \varphi \psi(\varphi, \theta) \phi^{*}\left(z^{*}\right) \mathrm{d} \varphi \mathrm{d} \theta \mathrm{d} z, \\
A_{e p 2} & =\pi \beta_{p} \int_{d^{*}+6 \delta_{b c}^{*}}^{d^{*}+110} \int_{0}^{2 \pi} \int_{0}^{\pi} A_{i e p 2}^{*} \sin \varphi \psi(\varphi, \theta) \phi^{*}\left(z^{*}\right) \mathrm{d} \varphi \mathrm{d} \theta \mathrm{d} z, \\
A_{p} & =\pi \beta_{p} \int_{d^{*}+110 \delta_{b c}^{*}}^{\infty} \int_{0}^{2 \pi} \int_{0}^{\pi} A_{i p}^{*} \sin \varphi \psi(\varphi, \theta) \phi^{*}\left(z^{*}\right) \mathrm{d} \varphi \mathrm{d} \theta \mathrm{d} z,
\end{aligned}
$$

where $\beta$ and $\beta_{p}$ are elastic and plastic roughness parameters defined as $\beta=\pi R \sigma_{z}$ and $\beta_{p}=\pi\left(R-\omega_{c}\right) \sigma_{z}$.

The relations for the contact area are as follows:

$$
\begin{aligned}
A_{i e}^{*} & =\delta_{v f}^{*} \cos \varphi, \\
A_{i e p 1}^{*} & =\delta_{v f}^{*} \cos \varphi, \\
A_{i e p 2}^{*} & =1.19\left(\delta_{v f}^{*} \cos \varphi-1\right)^{1.1}, \\
A_{i p}^{*} & =\delta_{v f}^{*} \cos \varphi\left(\frac{\delta_{v f}^{*} \cos \varphi}{1.9}\right)^{B} .
\end{aligned}
$$

Similarly, for the total contact stiffness, we can write

$$
K_{t o t}=K_{e}-K_{e p 1}+K_{e p 2}+K_{p}
$$

where

$$
\begin{aligned}
& K_{e}=\eta A_{n} \int_{d^{*}}^{d^{*}+\delta_{b c}^{*}} \int_{0}^{2 \pi} \int_{0}^{\pi} K_{i e}^{*} \sin \varphi \psi(\varphi, \theta) \phi^{*}\left(z^{*}\right) \mathrm{d} \varphi \mathrm{d} \theta \mathrm{d} z, \\
& K_{e p 1}=\eta A_{n} \int_{d^{*}+\delta_{b c}^{*}}^{d^{*}+6 \delta_{b c}^{*}} \int_{0}^{2 \pi} \int_{0}^{\pi} K_{i e p 1}^{*} \sin \varphi \psi(\varphi, \theta) \phi^{*}\left(z^{*}\right) \mathrm{d} \varphi \mathrm{d} \theta \mathrm{d} z, \\
& K_{e p 2}=\eta A_{n} \int_{d^{*}+6 \delta_{b c}^{*}}^{d^{*}+110 \delta_{b c}^{*}} \int_{0}^{2 \pi} \int_{0}^{\pi} K_{i e p 2}^{*} \sin \varphi \psi(\varphi, \theta) \phi^{*}\left(z^{*}\right) \mathrm{d} \varphi \mathrm{d} \theta \mathrm{d} z, \\
& K_{p}=\eta A_{n} \int_{d^{*}+110 \delta_{b c}^{*}}^{\infty} \int_{0}^{2 \pi} \int_{0}^{\pi} K_{i p}^{*} \sin \varphi \psi(\varphi, \theta) \phi^{*}\left(z^{*}\right) \mathrm{d} \varphi \mathrm{d} \theta \mathrm{d} z, \\
& K_{i e}^{*}=\frac{3}{2} \sqrt{\delta_{v f}^{*} \cos \varphi+2\left(r_{i e}^{*}\right)^{2},} \\
& K_{i e p 1}^{*}=\sqrt{\left(1.6764\left(\delta_{v f}^{*} \cos \varphi-1\right)^{0.27}\right)^{2}+4.2426\left(r_{i e p A T}^{*},\right.}, \\
& K_{i p}^{*}= \sqrt{\left(\frac{3}{S}\right)^{2}+4.5\left(r_{i p A T}^{*}\right)^{2} \cdot}, \\
& 2.81\left(\delta_{v f}^{*} \cos \varphi-1\right)^{0.54}+2.25\left(r_{i e p A T}^{*}\right)^{2}\left(2-\frac{r_{i e p A T}^{*} \sin \varphi\left(\zeta_{v}^{*} \sin \varphi+\xi_{v}^{*} \cos \varphi\right)}{\mu_{e p} F_{i n-e p}^{*}}\right)
\end{aligned}
$$




\section{Results and Discussion}

In this paper, a MATLAB program is employed to assess the outcomes of the suggested theoretical model to determine the contact problem of the rough surfaces considering asperities effects. The rough surfaces' properties assume to be constant, and the contact properties like the contact stiffness, which is very important in many applied problems such as wheel and rail interaction, are studied.

In this paper, in order to compare the obtained results with the results of the other works, the contact properties of the Gao model [19] are used. Therefore, $E=116 \mathrm{GPa}$, $G=41 \mathrm{GPa}, v_{1}=v_{2}=0.31, H=1.12 \mathrm{GPa}, S=0.58, \psi=6$, and $A_{n}=3 \times 10-5 \mathrm{~m}^{2}$ are considered, and the following results are attained.

Considering the presented model, Figure 4 shows the total contact force variation versus mean separation for different surface parameters $\sigma_{z}$. This figure demonstrates that the total contact force diminishes with growing separation, which is acceptable in real contact situations. Furthermore, whenever the surface parameter $\sigma$ lessens, the contact force decreases with a higher rate. This fact shows that, in the smooth surfaces, the contact force reduces at a higher rate in comparison to a rough one with rising separation, which is logical since the figures of asperities in contact on a smooth surface decrease at a higher rate than a rough one.

In Figure 5, the total contact force variation versus separation for different models is shown (the presented model is called the "AT model").

As is seen in Figure 5, the contact force of the AT model is slightly lesser than the other models. However, its trend of variation shows suitable concurrence with the other models. In the AT model, the effects of the slip-stick area are considered in the formulation, and in this way, the slight diversity between the AT model and the other models may be explained.

Considering the AT model, the real contact area variations versus mean separation for different surface parameters are shown in Figure 6. The results indicate that the real contact area has a reducing trend by rising mean separation, which is logical because the figure of asperities in contact diminishes with growing separation.

In Figure 6, considering a smoother surface (i.e., with smaller $\sigma_{z}$ ), the real contact area decreases at a higher rate with increasing separation in comparison to a rougher one, because the figures of asperities in contact on a smooth surface reduce at a higher rate with increasing separation in comparison to a rough one.

The real contact area variations versus mean separation for different models are shown in Figure 7. The effects of considered fictitious asperity in the elastic-plastic zone and its subtraction from the total results and some considered corrections in the AT model like $r_{i e p A T}$ and $\beta_{p}$ cause the results of the real contact area in this model to be lesser than the other models. At bigger separations, where the plastic region diminishes, the results are closer.

The variations of the contact stiffness versus the mean separation and the effects of the surface parameter $\sigma_{z}$ on the

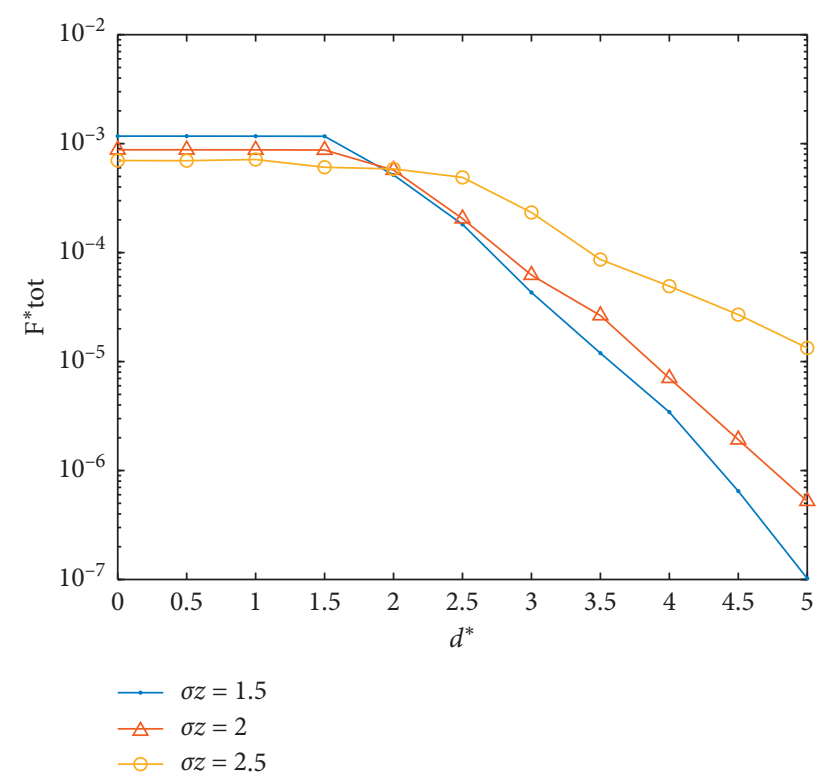

FIGURE 4: Variations of the total contact force with separation for different surface parameters.

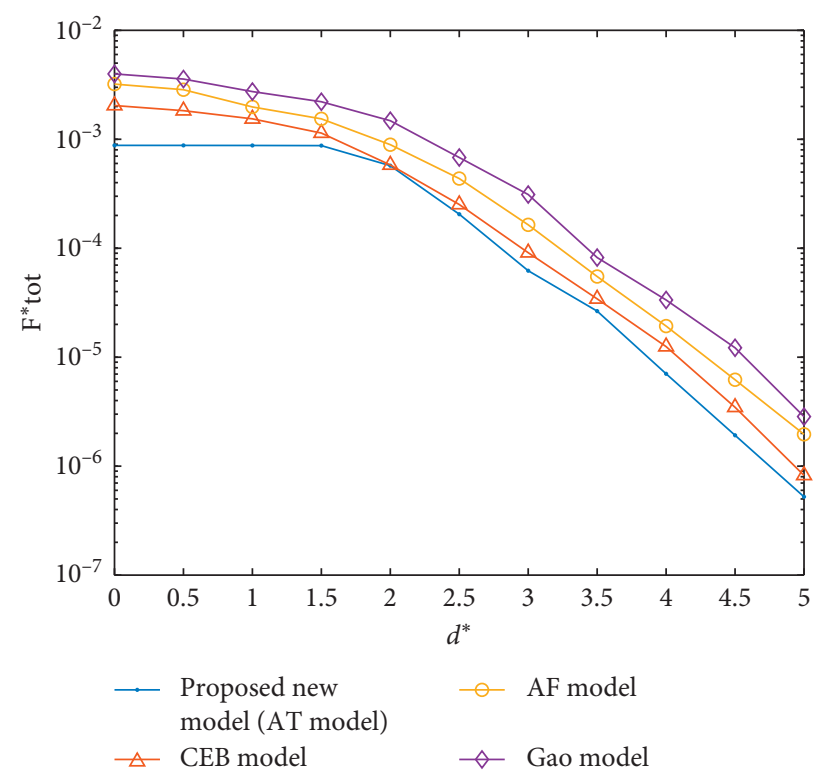

FIgURE 5: Total contact force variation versus separation for different models.

contact stiffness are shown in Figure 8. As is seen from this figure in lower separation, the contact stiffness rises.

This trend can be explained by the hardening process, which occurs in contacting asperities or the connection which exists between the mean separation and the real contact area. Figure 8 demonstrates that the contact stiffness of smoother surfaces rises at a higher rate than the rougher one with diminishing separation. This is logical since the real contact area of the smoother surface rises at a higher rate than the rougher one with diminishing separation.

Contact stiffness variations versus mean separation for different models are shown in Figure 9. As can be seen from 


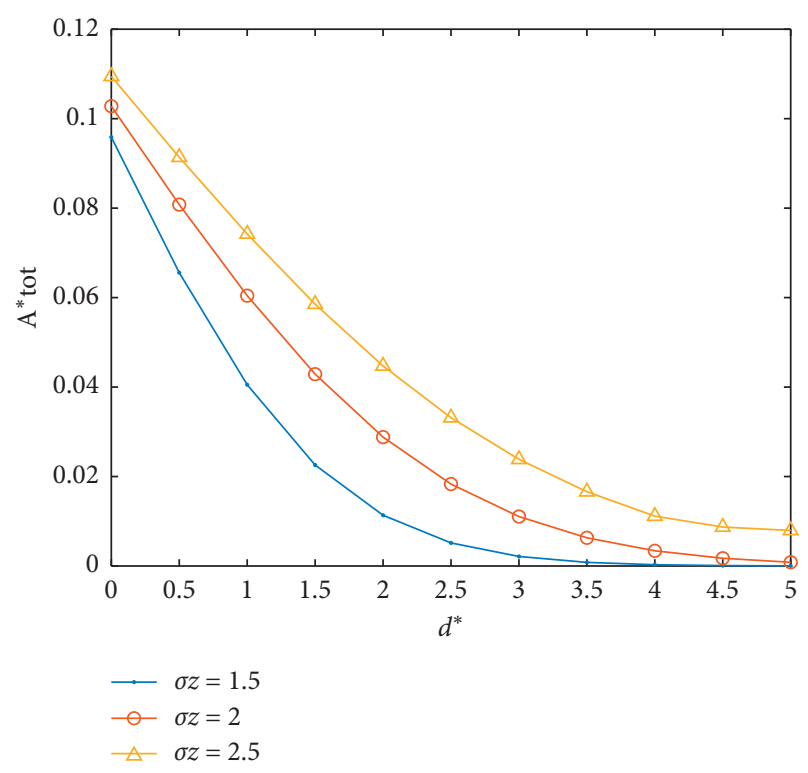

FIgURE 6: Real contact area variations versus mean separation for different surface parameters.

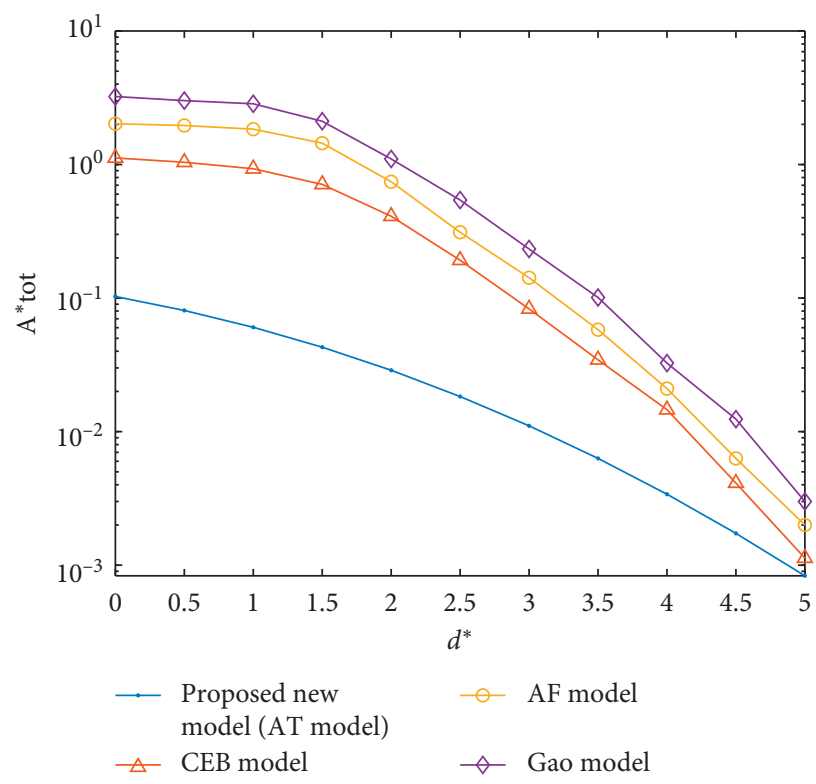

Figure 7: Variation of the contact area versus separation for different models.

this figure, the variation trend of the AT model results shows good agreement with other recognized models.

In Figure 10 the results of the contact area variations versus mean separation considering the experimental results, based on Kucharski et al [17] and other models, are compared. The material properties of the steel specimens considered in the experimental work are as follows: Young's modulus $E=200 \mathrm{GPa}$, Poisson's ratio $v=0.3$, and tensile yield strength $Y=400 \mathrm{MPa}$. The specimens are made of carbon steel with $0.45 \%$ of carbon.

In [17], measuring of contact load and the contact area is performed by the test, and sand-blasting is used to produce

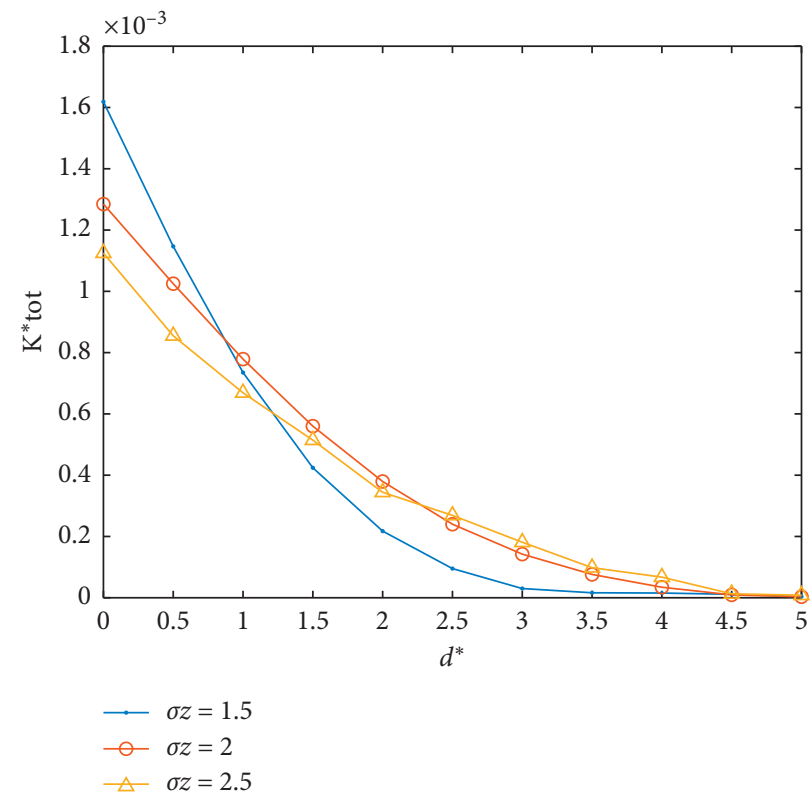

FIGURE 8: Variation of the contact stiffness versus the mean separation.

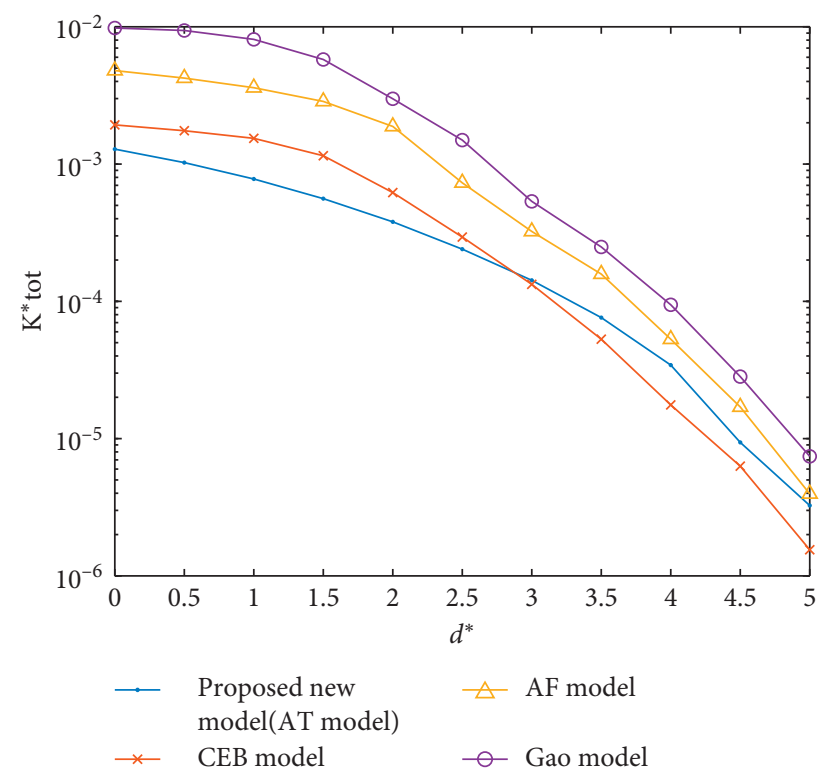

FIgURE 9: Contact stiffness variation versus mean separation for different models.

the surface roughness of specimens. The loadings of the surfaces are performed incrementally in a few steps up to $300 \mathrm{MPa}$ of nominal load. After each step of loading, the 3D topography of the surface is mapped and areas of plastic deformation are identified in the isometric view. The real contact area is measured by summarizing the areas of individual contact spots.

According to Figure 10, the results of the AT model rather than other analytical models are very close to the experimental results. Since in employed formulations of this model, the slip-stick effects are considered in elastic-plastic 


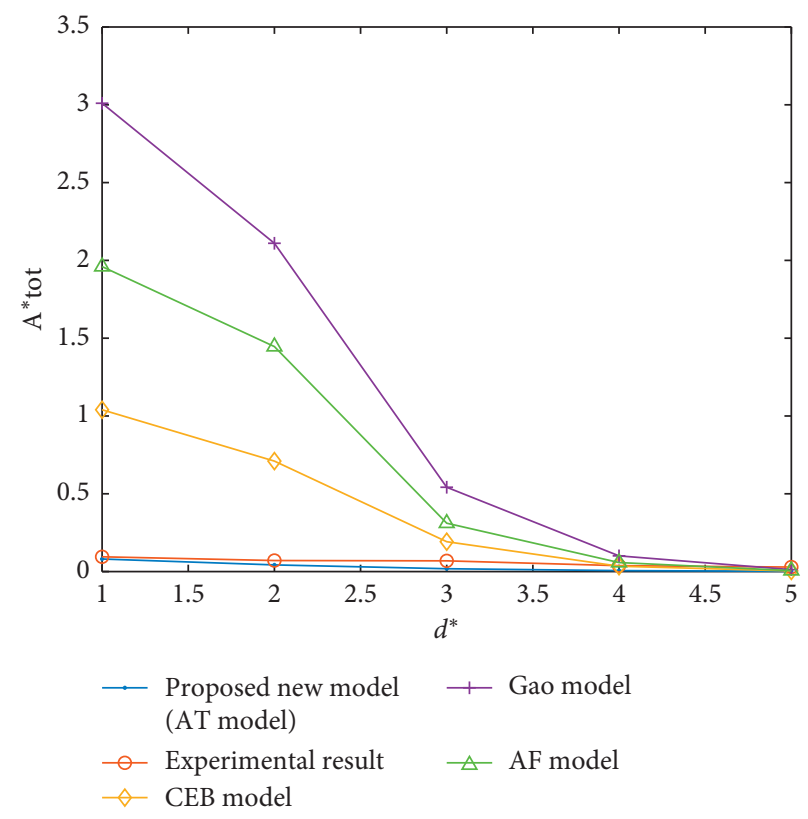

Figure 10: Contact area variations versus mean separation considering the experiment and other models.

zones and corrections on the modified plastic radius and plastic roughness parameters are performed.

In comparison to the experimental results, the error of the presented model at low mean separation and where the effects of plasticity are maximized is about $14 \%$. However, for the other models, this error is too high, and for bigger mean separation, where the plastic region diminishes, all the results are closed to each other. On the other hand, the AT model offers a worthy assessment for the asperities contact problem, especially at the low contact area and separation where high contact pressure and plastic deformation usually exist.

\section{Conclusions}

In this task, the effects of the surface asperities on the contact force, contact area, and contact stiffness are studied. The lateral contact between asperities is assumed to be the general condition for contacting surfaces. The Gao model is used for the fundamental of formulations, and corrections for the elastic-plastic contact zone, fictitious asperity theory, and slip-stick effects are carried out. Also, a new harmonic spherical distribution function for applying the contact angle effects is introduced, and a corrected radius for plastic deformation is obtained. From the achieved results and performed discussion, the following major outcomes are derived:

(1) In the contact of rough surfaces, total contact force, real contact area, and contact stiffness decrease with increasing the mean separation. These parameters increase at a higher rate with decreasing mean separation on a smoother surface.

(2) The outcomes of the AT model are close to the other available analytical models. The deviations in some results, especially where the plastic deformation is greater, are due to the effects of the considered parameters like the effects of the slip-stick in the elastic-plastic formulation and corrections in some important parameters like plastic radiuses and plastic roughness.

(3) Because of considering the effects of slip-stick in the elastic-plastic regime, performing some modifications in plastic region parameters such as plastic radius, roughness parameter, formulations of $F_{e p 1}$, $A_{e p 1}$, and $K_{e p 1}$, and using a new harmonic spherical distribution function for contact angles effects, the results of the AT model, in comparison with the other considered models with high accuracy, are near to the experimental results, especially in a lower separation where the plastic deformation is dominated.

(4) In comparison to the experimental results, the error of the AT model considering low mean separation is about $14 \%$. While for the other models, this error is too high. For greater mean separation, where the plastic region diminishes, all results are approximately close to each other. On the other hand, the results of the presented model offer very good estimations for the asperities contact characteristics, especially at the small-contact area and separation where high-contact pressure and plastic deformation usually exist.

In future works, the presented model can be used to simulate the applied problems with high accuracy. For example, in the wheel-rail contact problem, various failures such as fracture and wear are prevalent that need to be expressed and analyzed with an accurate model.

\section{Nomenclature}

$A_{e}: \quad$ Elastic contact area

$A_{e p 1}$ : Elastic-plastic first component of contact area

$A_{e p 2}$ : Elastic-plastic second component of contact area

$A_{p}: \quad$ Plastic contact area

$A_{n}: \quad$ Nominal contact area

$A_{\text {tot }}: \quad$ Total contact area

$a_{k 0}: \quad$ Fabric factor

$a_{k m}: \quad$ Fabric factor

$b_{k m}: \quad$ Fabric factor

$d$ : Mean separation of two rough surfaces

E: $\quad$ Equivalent elastic modulus

$F_{i}$ : Resultant force

$F_{i n}: \quad$ Normal component of force

$F_{i \tau}$ : Tangential component of force

$F_{t o t}: \quad$ Total contact force

$F_{e}: \quad$ Elastic contact force

$F_{e p 1}$ : Elastic-plastic first component of contact force

$F_{e p 2}$ : Elastic-plastic second component of contact force

$F_{p}$ : Plastic contact force

$F_{\text {inc }}: \quad$ Critical value at the inception of plastic deformation

$G$ : $\quad$ Equivalent shear modulus

$H$ : $\quad$ Hardness of softer material 
$K_{t o t}: \quad$ Total contact stiffness

$K_{e}: \quad$ Elastic contact stiffness

$K_{e p 1}$ : Elastic-plastic first component of contact stiffness

$K_{e p 2}$ : Elastic-plastic second component of contact stiffness

$K_{p}: \quad$ Plastic contact stiffness

$Q_{p}$ : $\quad$ Property symbol related to fully plastic regime

$R: \quad$ Equivalent radius of asperities

$R_{p}: \quad$ Equivalent plastic radius

$r_{i(p) A T}^{*}$ : Nondimensional new radius of contact area at plastic zones

$r_{i(p)}^{*}$ : Nondimensional plastic or elastic-plastic radius of contact area

$r_{i c}: \quad$ Critical radius at the inception of plastic deformation

$r_{i e}^{*}: \quad$ Nondimensional elastic radius of the contact area between the contacting asperities

S: $\quad$ Hardness factor

$Z$ : $\quad$ Height of asperity measured from the mean asperity height

$\beta$ : $\quad$ Elastic roughness parameters

$\beta_{p}: \quad$ Plastic roughness parameters

$\delta_{b w}$ : Deformation caused by the interaction between the adjacent asperities

$\delta_{b c}^{*}: \quad$ Nondimensional deformation parameter

$\delta_{v f}^{*}$ : Nondimensional normal component of deformation in the $Z$-axis direction

$\zeta_{v}^{*}: \quad$ Nondimensional tangential component of displacement in the $\mathrm{Z}$-axis direction

$\eta$ : $\quad$ Area density of asperities

$\Phi: \quad$ Contact angle

$\theta$ : $\quad$ Azimuthal angle

$\mu_{e p}: \quad$ Friction factor at elastic-plastic contact deformations

$v$ : $\quad$ Poisson's ratio

$\xi_{v}^{*}: \quad$ Nondimensional horizontal component of displacement in the $Z$-axis direction

$\phi^{*}$ : Nondimensional Gaussian distribution function

$\sigma: \quad$ Standard deviation of the surface height distribution

$\sigma_{z}: \quad$ Standard deviation of asperity height distribution

$\psi: \quad$ Distribution function for asperity contacts

$\omega_{c}$ : Critical deformation at the inception of plastic

deformation

*: $\quad$ Nondimensional parameters.

\section{Data Availability}

The data supporting the current study are available from the corresponding author upon request.

\section{Conflicts of Interest}

The authors declare that they have no conflicts of interest.

\section{References}

[1] Y. Wu, Y. Wei, Y. Liu, Z. Duan, and L. Wang, "3-D analysis of thermal-mechanical behavior of wheel/rail sliding contact considering temperature characteristics of materials," Applied Thermal Engineering, vol. 115, pp. 455-462, 2017.

[2] P. Li, Y. Zhai, Q. Wang, W. Fu, and H. Yang, "Investigation of the contact performance of machined surface morphology," Tribology International, vol. 107, pp. 125-134, 2017.

[3] O. E. Lundberg, A. Nordborg, and I. Lopez Arteaga, "The influence of surface roughness on the contact stiffness and the contact filter effect in nonlinear wheel-track interaction," Journal of Sound and Vibration, vol. 366, pp. 429-446, 2016.

[4] J. A. Greenwood and J. B. P. Williamson, "Contact of nominally flat surfaces," Proceedings of the Royal Society A, vol. 295, no. 1442, pp. 300-319, 1966.

[5] W. R. Chang, I. Etsion, and D. B. Bogy, "An elastic-plastic model for the contact of rough surfaces," Journal of Tribology, vol. 109, no. 2, pp. 257-263, 1987.

[6] Y. Zhao, D. M. Maietta, and L. Chang, "An asperity microcontact model incorporating the transition from elastic deformation to fully plastic flow," Journal of Tribology, vol. 122, no. 1, pp. 86-93, 2000.

[7] L. Kogut and I. Etsion, "A finite element-based elastic-plastic model for the contact of rough surfaces," Modelling and Simulation in Engineering, vol. 2011, no. 3, pp. 383-390, Article ID 561828, 2011.

[8] I. Etsion, "A static friction model for elastic-plastic contacting rough,” ASME Journal of Tribology, vol. 126, no. 1, pp. 34-40, 2004.

[9] R. L. Jackson and I. Green, “A finite element study of elastoplastic hemispherical contact against a rigid flat," Journal of Tribology, vol. 127, no. 2, pp. 343-354, 2005.

[10] B. Zhao, S. Zhang, and Z. Qiu, "Analytical asperity interaction model and numerical model of multi-asperity contact for power hardening materials," Tribology International, vol. 92, pp. 57-66, 2015.

[11] M. Ciavarella, "Rough contacts near full contact with a very simple asperity model," Tribology International, vol. 93, pp. 464-469, 2015.

[12] P. Cinat, M. Paggi, and G. Gnecco, "Identification of roughness with optimal contact response with respect to real contact area and normal stiffness," Journal of Mathematical Problem in Engineering, vol. 2019, Article ID 7051512, 11 pages, 2019.

[13] Y. Wen, J. Tang, W. Zhou, and C. Zhu, "A new elliptical microcontact model considering elastoplastic deformation," Proceedings of the Institution of Mechanical Engineers, Part J: Journal of Engineering Tribology, vol. 232, no. 11, pp. 13521364, 2018.

[14] O. M. Abuzeid, A. N. Al-Rabadi, and H. S. Alkhaldi, "Recent advancements in fractal geometric-based nonlinear time series solutions to the micro-quasistatic thermoviscoelastic creep for rough surfaces in contact," Journal of Mathematical Problem in Engineering, vol. 2011, Article ID 691270, 29 pages, 2011.

[15] A. Megalingam and K. S. Hanumanth Ramji, "A complete elastic-plastic spherical asperity contact model with the effect of isotropic strain hardening," Journal of Engineering Tribology, vol. 20, 2020.

[16] J. Abdo and K. Farhang, "Elastic-plastic contact model for rough surfaces based on plastic asperity concept," International Journal of Non-linear Mechanics, vol. 40, no. 4, pp. 495-506, 2005.

[17] S. Kucharski, T. Klimczak, A. Polijaniuk, and J. Kaczmarek, "Finite-elements model for the contact of rough surfaces," Wear, vol. 177, no. 1, pp. 1-13, 1994. 
[18] W.-R. Chang, "An elastic-plastic contact model for a rough surface with an ion-plated soft metallic coating," Wear, vol. 212, no. 2, pp. 229-237, 1997.

[19] Z. Gao, W. Fu, W. Wang, W. Kang, and Y. Liu, "The study of anisotropic rough surfaces contact considering lateral contact and interaction between asperities," Tribology International, vol. 126, pp. 270-282, 2018.

[20] C. Cattaneo, "Sulcontatto di due corpielastici: distribuzione locale deglisforzi," Rendiconti Dell' AecademiaNazionaledeiLincei, vol. 27, pp. 342-348, 1938.

[21] R. D. Mindlin and H. Deresiewicz, "Elastic spheres in contact under varying oblique forces," ASME Journal of Applied Mechanics, vol. 20, pp. 327-344, 1953.

[22] M. Eriten, A. A. Polycarpou, and L. A. Bergman, "Physicsbased modeling for fretting behavior of nominally flat rough surfaces," International Journal of Solids and Structures, vol. 48, no. 10, pp. 1436-1450, 2011.

[23] P. Poorsolhjouy and M. Gonzalez, "Connecting discrete particle mechanics to continuum granular micromechanics: anisotropic continuum properties under compaction," $M e$ chanics Research Communications, vol. 92, pp. 21-27, 2018.

[24] J. Jäger, "Axi-symmetric bodies of equal material in contact under torsion or shift," Archive of Applied Mechanics, vol. 65, no. 7, pp. 478-487, 1995. 